\title{
Tobacco Stalk Biochar Application Improves Soil Fertility and Flue- Cured Tobacco Growth
}

\author{
Yan $\mathrm{Li}^{1 \dagger}$, Tianxiang $\mathrm{Liu}^{1,2 \dagger}$, Xian $\mathrm{He}^{1 \dagger}$, Rui Liu ${ }^{1}$, Tianyang $\mathrm{Xu}^{1}$, Mengyang $\mathrm{Hu}^{1}$, Kaiyuan $\mathrm{Gu}^{1}$, Jiaen $\mathrm{Su}^{1^{*}}$ and \\ Congming Zou ${ }^{1 *}$ \\ ${ }^{1}$ Yunnan Academy of Tobacco Agricultural Sciences, 33 Yuantong Street, Kunming, Yunnan 650021, P. R. China \\ ${ }^{2}$ College of Tobacco Science, Yunnan Agricultural University, Kunming, Yunnan 650201, P. R. China \\ *For correspondence: zoucongmingzcm@163.com; 705763042@qq.com \\ ${ }^{\dagger}$ Contributed equally to this work and are co-first authors \\ Received 14 October 2020; Accepted 27 November 2020; Published 25 January 2021
}

\begin{abstract}
Tobacco stalks the main agricultural waste after tobacco harvest, are generally discarded directly or returned to the field after burning. They are rarely processed into biochar, a product that could benefit soil properties. To explore the effects of applying tobacco stalk biochar on soil fertility and tobacco production, tobacco was grown at six biochar application levels $(0,3,000$, 4,500, 6,000, 9,000 and 12,000 $\mathrm{kg} \mathrm{ha}^{-1}$ ) in three different sites (Jianchuan, Midu and Eryuan) in Dali County, Yunnan Province. Biochar decreased soil bulk density, increased large and small soil aggregate proportion, and increased soil organic carbon and nitrogen stocks. Biochar also improved the yield and quality of tobacco leaves at all sites. Biochar rates of 3,000, 4,500 and $6,000 \mathrm{~kg} \mathrm{ha}^{-1}$ linearly improved soil fertility and agronomic traits while application rate of biochar exceeding 9,000 $\mathrm{kg} \mathrm{ha}^{-1}$ reduced plant growth. Moreover, the optimum biochar application rates for better plant height, stem diameter, maximum leaf length and leaf width, yield, and average price differed by site. These rates were: $6,000 \mathrm{~kg} \mathrm{ha}^{-1}$ (Midu), 3,000

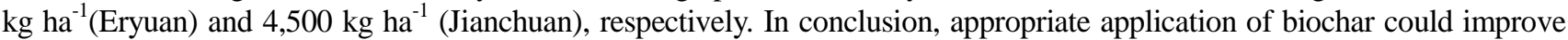
soil nutrients and contribute to tobacco growth in different soil nutrient conditions. (C) 2021 Friends Science Publishers
\end{abstract}

Keywords: Tobacco stalk; Biochar; Soil quality; Flue-cured tobacco; Growth

\section{Introduction}

Tobacco (Nicotiana tobacum L.) the most important economic crop in southwest regions of China, reached a cultivated area of 53, 0000 ha in 2019 (Zhang and Ma 2020). To achieve higher economic benefits and yield, tobacco growers usually apply large amounts of chemical fertilizer and practice continual tobacco monocropping. This causes constant loss of soil nutrients and deterioration of soil physical and chemical properties, leading to soil compaction, soil nutrient imbalance, soil microflora change, and frequent occurrence of soil-borne diseases (Cheng et al. 2013; Qin et al. 2015; Ma et al. 2017).

Tobacco stalks after leaf harvest are agricultural waste, with a biomass of $2250 \sim 3000 \mathrm{~kg} \mathrm{ha}^{-1}$. There are about 3 million metric tons of tobacco stalks in China's tobacco areas (Han and Wang 2016). In traditional management, most of these stalks are burned and returned to the field as ash because fresh stalks and roots left in field could cause disease for the next tobacco growing season (Zou et al. 2017). However, this management is resource wasteful and pollutes the environment. Balancing waste tobacco stalk production and use with continually decreased soil quality is a challenge and opportunity for sustainable tobacco production.

Biochar is a term for the solid material formed by pyrolysis or carbonization of biomass such as plant tissue, agricultural, and forestry residues, plant straw, etc. (ElNaggar et al. 2019). The typical carbon content of biochar is $65-90 \%$, and it contains $3-20 \%$ of potassium, phosphorus, calcium, magnesium, silicon, manganese, zinc and other oxides and nitrogen compounds (Emma 2006; Yuan et al. 2016; Li et al. 2017). Biochar has good water absorption and adsorption capacity because of its large intermolecular distance (Wang and Sun 2016; Hussain et al. 2017; Farooq et al. 2020a). After application, biochar can loosen the soil increase the number of aggregates in soil, and increase the soil pH (Lin et al. 2018; Li et al. 2019; Farooq et al. 2020b). The nutrient elements in biochar could increase the content of organic matter in soil and reduce nitrogen leaching (Sarma et al. 2018; Zhang et al. 2018; Wei et al. 2019; Baruah et al. 2020; Liu et al. 2020). Biochar can reduce or inhibit soil borne diseases, promote carbon and nitrogen metabolism, and improve crop yield and quality even under less than optimum conditions (Wang et al. 2015; Farooq et

To cite this paper: Li Y, T Liu, X He, R Liu, T Xu, M Hu, K Gu, J Su, C Zou (2021). Tobacco stalk biochar application improves soil fertility and flue-cured tobacco growth. Intl J Agric Biol 25:632-638 
al. 2020c).

Reports on biochar have mainly focused on reducing heavy metals in soil, remediation of soil pollution, soil nutrients, and the microbial community. There are few reports about using tobacco stalk biochar to influence soil aggregate structure and soil organic carbon and nitrogen stocks during tobacco growth. Three tobacco growing sites with different nutrient status were selected to explore the proportion of different particle size aggregates, the changes of organic carbon and soil nitrogen stocks, and tobacco yield and quality. Exploring the influence of tobacco stalk biochar on soil nutrients and plant growth would support sustainable and recyclable agricultural management in tobacco growing regions.

\section{Materials and Methods}

\section{General situation of test sites}

This study was conducted at three sites: Midu, Eryuan and Jianchuan, Dali City, Yunnan Province, China. The basic geographical conditions and soil physical and chemical properties in the three sites are in Tables 1 and 2. Sampling and measurement in this study occurred from April to September 2017.

\section{Experiment design}

Six different biochar levels were used at each site: $0,3,000$, $4,500,6,000,9,000$, and $12,000 \mathrm{~kg} \mathrm{ha}^{-1}$. Biochar was produced from tobacco stalks by combustion in an open-top carbonization device under hypoxic condition at about $450^{\circ} \mathrm{C}$ for $30 \mathrm{~min}$. The basic physico-chemical properties of tobacco stalk biochar are in Table 3 .

The study sites were initially established in 2016, and sampling started in 2017. The experiment followed a randomized complete block design with factorial arrangement and was replicated three times with plot size of $14 \mathrm{~m} \times 2 \mathrm{~m}$. The biochar was scattered onto the soil surface by hand and then ploughed to achieve mixing to $40 \mathrm{~cm}$ depth soil. A flue-cured tobacco variety, 'K326,' was used as plant material. The planting density was 16,500 plants ha ${ }^{-1}$, and plant spacing was $50 \mathrm{~cm} \times 120 \mathrm{~cm}$. A compound fertilizer $\left(\mathrm{N}: \mathrm{P}_{2} \mathrm{O}_{5}: \mathrm{K}_{2} \mathrm{O}=1: 2: 2.5\right)$ amounting $75 \mathrm{~kg} \mathrm{ha}^{-1}$ was strip-applied, with one third applied as a base fertilizer before transplanting and the remaining two thirds applied 30 days after transplanting.

\section{Soil sampling and sample preparation}

Soil samples were collected from April to September. Soil bulk density (BD) was measured with a cutting ring with a volume of $100 \mathrm{~cm}^{3}$. The particle size distribution of aggregates in soil, soil organic carbon stocks (SOCs), and total soil nitrogen stocks (TSNs) were determined by separate random sampling to obtain
54 soil samples from depths of $0 \sim 20 \mathrm{~cm}$ and $20 \sim 40 \mathrm{~cm}$ in each block. After removing roots and pebbles, the soil was placed in self-sealing plastic bags, and stored in a refrigerator at $4^{\circ} \mathrm{C}$. Within a week, the soil aggregates were screened by wet sieving method.

\section{Sieving of wet aggregates}

To avoid disruption during rewetting of dried soil fieldmoist soil was used to size grades within the aggregate samples (Zou et al. 2015).

The mean weight diameter (MWD) and the geometric mean diameter (GMD) of the aggregates, the SOC or TSN stock in each aggregate size fraction and whole soil were computed using Equation (1), (2), (3), and (4), respectively (Zou et al. 2015).

$$
\begin{gathered}
\text { MWD }=\sum_{i=1}^{n} X i \times w i \\
\text { GMD }=\exp \left[\frac{\sum_{i=1}^{n} \text { WixLnXi }}{\sum_{i=1}^{n} \text { Wi }}\right] \\
\text { SOCs (or TSNs) }=\mathrm{D} \times \mathrm{BD} \times \operatorname{SOC}(\text { or TSNi) } \times W i \\
\text { SOCsi (or TSNsi) }=\frac{\text { DXBDXSOCi (or TSNi) } \times W i}{10}
\end{gathered}
$$

Agronomic traits were determined according to the Investigating and Measuring Methods of Agronomical Character of Tobacco in Tobacco Industry Standard of the People's Republic of China YC/T142-2010. Fifteen fluecured tobacco plants from each plot were evaluated in the rosette stage.

Yield refers to the total yield of tobacco leaves per unit area. The $5 \sim 8^{\text {th }}$ leaves (lower leaves), 10 13 ${ }^{\text {th }}$ leaves (middle leaves), and 14 17 $7^{\text {th }}$ leaves (upper leaves) were collected from the bottom to the top of tobacco stalk position when the tobacco leaves were mature. After fluecuring, the tobacco samples were graded and measured according to Chinese Standard GB 2635-1992, and indices, such as proportions (\%) of top and middle-grade tobacco were obtained. The output was calculated according to the purchase price for that year (2017).

\section{Data analysis}

Data were analyzed with the General Linear Model (GLM) procedure of the S.A.S. 9.3 computer package (S.A.S. Institute Inc., Cary, NC). This study used by factor design including study sites and biochar application rates. There were significant treatment effects if the probability $(P)$ was $<0.05$. Sigma Plot 14.0 (Systat Software Inc., Chicago, IL, USA) was used to produce all associated output plots.

\section{Results}

\section{Effects of biochar on soil physical properties}

When tobacco stalk biochar application rates were more than $4500 \mathrm{~kg} \mathrm{ha}^{-1}$, there were significant decreases in soil 
Table 1: Basic geographical conditions of different test sites

\begin{tabular}{|c|c|c|c|c|c|}
\hline & Longitude and latitude & Altitude $(\mathrm{m})$ & Annual average temperature $\left({ }^{\circ} \mathrm{C}\right)$ & Annual rainfall $(\mathrm{mm})$ & Annual sunshine hours (h) \\
\hline Jianchuan & $\mathrm{N} 24^{\circ} 47^{\prime}, \quad$ E $100^{\circ} 19^{\prime}$ & 1780 & 18.3 & 824.4 & 2750 \\
\hline Midu & $\mathrm{N} 25^{\circ} 23^{\prime}, \quad$ E $100^{\circ} 16^{\prime}$ & 1980 & 16.3 & 665.6 & 2740 \\
\hline Eryuan & $\mathrm{N} 28^{\circ} 56^{\prime}, \quad$ E $99^{\circ} 13^{\prime}$ & 1590 & 20.0 & 559.4 & 2720 \\
\hline
\end{tabular}

Table 2: Physical and chemical properties of soil foundation

\begin{tabular}{|c|c|c|c|c|c|c|c|c|}
\hline Sites & $\begin{array}{l}\mathrm{pH} \\
(2.5: 1)\end{array}$ & $\begin{array}{l}\text { Soil organic } \\
\text { matter }(\mathrm{g} / \mathrm{kg})\end{array}$ & $\begin{array}{l}\text { Total nitrogen } \\
(\mathrm{g} / \mathrm{kg})\end{array}$ & $\begin{array}{l}\text { Total phosphorus } \\
(\mathrm{g} / \mathrm{kg})\end{array}$ & $\begin{array}{l}\text { Total potassium } \\
(\mathrm{g} / \mathrm{kg})\end{array}$ & $\begin{array}{l}\text { Hydrolyzable } \\
\text { nitrogen }(\mathrm{g} / \mathrm{kg})\end{array}$ & $\begin{array}{l}\text { Available } \\
\text { phosphorus }(\mathrm{g} / \mathrm{kg})\end{array}$ & $\begin{array}{l}\text { Available } \\
\text { potassium }(\mathrm{g} / \mathrm{kg})\end{array}$ \\
\hline Midu & 6.5 & 56.2 & 2.8 & 1.1 & 17.6 & 210.8 & 91.3 & 285.5 \\
\hline Eryuan & 6.7 & 27.4 & 1.3 & 0.9 & 10.4 & 154.3 & 36.7 & 62.4 \\
\hline
\end{tabular}

Table 3: The physical and chemical characteristics of tobacco stalk biochar in this study

\begin{tabular}{|c|c|c|c|c|c|c|c|c|c|}
\hline $\begin{array}{l}\text { Gravimetric Water } \\
\text { content }(\%)\end{array}$ & $\begin{array}{l}\text { Carbon } \\
\text { content }(\%)\end{array}$ & $\begin{array}{l}\text { Ash content } \\
(\%)\end{array}$ & $\begin{array}{l}\text { Specific surface } \\
\text { area }\left(\mathrm{m}^{2} \cdot \mathrm{g}^{-1}\right)\end{array}$ & $\mathrm{pH}$ & $\begin{array}{l}\text { Total } \\
\text { nitrogen }(\%)\end{array}$ & $\begin{array}{l}\text { Mineralizable } \\
\text { nitrogen }\left(\mathrm{mg} \mathrm{kg}^{-1}\right)\end{array}$ & $\begin{array}{l}\text { Available phosphorus } \\
\left(\mathrm{mg} \mathrm{kg}^{-1}\right)\end{array}$ & $\begin{array}{l}\text { Available } \\
\text { potassium (\%) }\end{array}$ & $\begin{array}{l}\text { Chloride } \\
\text { content }(\%)\end{array}$ \\
\hline 6.5 & 66.7 & 18.3 & 6.0 & 7.3 & 3.4 & 42.5 & 227 & 1.3 & 0.1 \\
\hline
\end{tabular}

Table 4: The effect of biochar application on soil bulk density (BD), the mean weight diameter (MWD), and the geometric mean diameter (GMD) in $0-20 \mathrm{~cm}$ and $20-40 \mathrm{~cm}$

\begin{tabular}{|c|c|c|c|c|c|c|c|c|c|c|}
\hline \multirow[t]{2}{*}{ Depth $(\mathrm{cm})$} & \multirow[t]{2}{*}{ Biochar levels $\left(\mathrm{kg} \mathrm{ha}^{-1}\right)$} & \multicolumn{3}{|c|}{ Bulk density (BD) $\left(\mathrm{g} / \mathrm{cm}^{3}\right)$} & \multicolumn{3}{|c|}{ MWD (mm) } & \multicolumn{3}{|c|}{ GMD (mm) } \\
\hline & & Midu & Eryuan & Jianchuan & Midu & Eryuan & Jianchuan & Midu & Eryuan & Jianchuan \\
\hline \multirow[t]{5}{*}{$0-20$} & 0 & $1.2 \mathrm{~b}$ & $1.2 \mathrm{~b}$ & $1.3 \mathrm{a}$ & $1.7 \mathrm{i}$ & $1.8 \mathrm{hi}$ & $1.8 \mathrm{~g}-\mathrm{i}$ & $0.5 \mathrm{j}$ & $0.5 \mathrm{j}$ & $0.5 \mathrm{j}$ \\
\hline & 3000 & $1.2 \mathrm{~b}$ & $1.2 \mathrm{~b}$ & $1.2 \mathrm{~b}$ & $1.8 \mathrm{f}-\mathrm{i}$ & $2.0 \mathrm{~d}-\mathrm{h}$ & $2.0 \mathrm{~d}-\mathrm{g}$ & $0.6 \mathrm{~h}-\mathrm{j}$ & $0.7 \mathrm{~g}-\mathrm{i}$ & $0.6 \mathrm{~h}-\mathrm{j}$ \\
\hline & 6000 & $1.2 \mathrm{~b}$ & $1.2 \mathrm{~b}$ & $1.2 \mathrm{~b}$ & $2.0 \mathrm{c}-\mathrm{f}$ & $2.1 \mathrm{c}-\mathrm{e}$ & $2.3 \mathrm{ab}$ & $0.8 \mathrm{c}-\mathrm{f}$ & $0.8 \mathrm{c}-\mathrm{f}$ & $0.9 \mathrm{~b}-\mathrm{d}$ \\
\hline & 9000 & $1.2 \mathrm{~b}$ & $1.2 \mathrm{~b}$ & $1.2 \mathrm{~b}$ & $2.1 \mathrm{c}-\mathrm{e}$ & $2.2 \mathrm{a}-\mathrm{c}$ & $2.4 \mathrm{a}$ & $0.9 \mathrm{c}-\mathrm{e}$ & $0.9 \mathrm{a}-\mathrm{c}$ & $1.1 \mathrm{ab}$ \\
\hline & 12000 & $1.2 \mathrm{~b}$ & $1.2 \mathrm{~b}$ & $1.2 \mathrm{~b}$ & $2.1 \mathrm{~b}-\mathrm{d}$ & $2.3 \mathrm{a}$ & $2.4 \mathrm{a}$ & $0.9 \mathrm{~b}-\mathrm{d}$ & $1.1 \mathrm{a}$ & $1.1 \mathrm{a}$ \\
\hline \multirow[t]{6}{*}{$20-40$} & 0 & $1.2 \mathrm{~b}$ & $1.3 \mathrm{a}$ & $1.2 \mathrm{~b}$ & $2.0 \mathrm{~h}-\mathrm{j}$ & $1.6 \mathrm{k}$ & $1.9 \mathrm{ij}$ & $0.6 \mathrm{gh}$ & $0.4 \mathrm{i}$ & $0.4 \mathrm{hi}$ \\
\hline & 3000 & $1.2 \mathrm{~b}$ & $1.3 \mathrm{a}$ & $1.2 \mathrm{~b}$ & $2.2 \mathrm{ef}$ & $1.8 \mathrm{ij}$ & $2.2 \mathrm{e}-\mathrm{g}$ & 0.7 ef & $0.5 \mathrm{~g}-\mathrm{i}$ & $0.6 \mathrm{gh}$ \\
\hline & 4500 & $1.2 \mathrm{~b}$ & $1.2 \mathrm{~b}$ & $1.2 \mathrm{~b}$ & $2.4 \mathrm{c}-\mathrm{e}$ & $1.9 \mathrm{ij}$ & $2.3 \mathrm{c}-\mathrm{f}$ & $0.9 \mathrm{~cd}$ & $0.6 \mathrm{gh}$ & 0.7 ef \\
\hline & 6000 & $1.2 \mathrm{~b}$ & $1.2 \mathrm{~b}$ & $1.2 \mathrm{~b}$ & $2.4 \mathrm{~b}-\mathrm{d}$ & $2.0 \mathrm{~g}-\mathrm{i}$ & $2.4 \mathrm{~b}-\mathrm{d}$ & $1.0 \mathrm{bc}$ & $0.6 \mathrm{fg}$ & $0.8 \mathrm{~d}-\mathrm{f}$ \\
\hline & 9000 & $1.2 \mathrm{~b}$ & $1.2 \mathrm{~b}$ & $1.2 \mathrm{~b}$ & $2.5 \mathrm{a}-\mathrm{c}$ & $2.2 \mathrm{f}-\mathrm{h}$ & $2.6 \mathrm{ab}$ & $1.1 \mathrm{ab}$ & $0.8 \mathrm{de}$ & $0.9 \mathrm{~cd}$ \\
\hline & 12000 & $1.2 \mathrm{~b}$ & $1.2 \mathrm{~b}$ & $1.2 \mathrm{~b}$ & $2.6 \mathrm{ab}$ & $2.3 \mathrm{~d}-\mathrm{f}$ & $2.7 \mathrm{a}$ & $1.2 \mathrm{a}$ & $0.9 \mathrm{~cd}$ & $1.2 \mathrm{a}$ \\
\hline
\end{tabular}

Means with different lowercase letters, within a column and rows for each trait, are statistically different from each other at $P<0.05$ according to DNMR test

bulk density (BD) depending on site and depth (Table 4). At 0-20 cm depth, when biochar application rate reached 4500 $\mathrm{kg} \mathrm{ha}^{-1}$, the MWD and GMD in each site were significantly higher than the control. At $6000 \mathrm{~kg} \mathrm{ha}^{-1}$, the MWD became significantly different between sites (Table 4). At $20-40 \mathrm{~cm}$ depth, the effect of biochar on increasing MWD and GMD was more obvious than at 0-20 $\mathrm{cm}$ depth (Table 4).

Biochar to 4,500 or $6,000 \mathrm{~kg} \mathrm{ha}^{-1}$ significantly increased the large macroaggregate proportion (LMP) and small macroaggregate proportion (SMP) in each site relative to the control and this trend at $20-40 \mathrm{~cm}$ depth was more obvious than that at $0-20 \mathrm{~cm}$ depth (Table 5). The SMP of each site increased significantly at 4,500 $\mathrm{kg} \mathrm{ha}^{-1}$ biochar application with site specific differences at $20-40 \mathrm{~cm}$ depth. Biochar application reduced soil MIP and SCP in each site. At $0-20 \mathrm{~cm}$ depth, when the biochar rate was $4500 \mathrm{~kg} \mathrm{ha}^{-1}$, the soil MIP began to be significantly lower than the control. In the $20-40 \mathrm{~cm}$ depth, there were significant site differences in MIP. The SCP in soil was significantly reduced relative to the control when the biochar application rate was $3,000 \mathrm{~kg} \mathrm{ha}^{-1}$. At $20-40 \mathrm{~cm}$ soil depth, there were significant site differences in SCP.

Biochar could significantly increase SOCs and TSNs (Table 6). The SOCs in 0-20 cm depth at Midu increased most obviously (40-50\%), followed by Eryuan and Jianchuan. Among three sites, the SOCs in Jianchuan were significantly less than in Midu and Eryuan. At $20-40 \mathrm{~cm}$ depth, the SOCs in Midu was significantly higher than in Eryuan and Jianchuan. Unlike SOCs, biochar addition most increased TSNs in Jianchuan at 0-20 cm depth (41-110\%), followed by Midu and Eryuan. The TSNs in Midu were also significantly higher than in Jianchuan after biochar application.

\section{Effects of biochar on agronomic traits of tobacco}

Compared with the control, when the biochar application rate was 6,000 and $12,000 \mathrm{~kg} \mathrm{ha}^{-1}$, plant height of fluecured tobacco significantly increased. When the biochar rate was $9,000 \mathrm{~kg} \mathrm{ha}^{-1}$, plant height was significantly lower than the control (Table 7). After applying biochar, plant height of flue-cured tobacco in Eryuan was 
Biochar Application to Improve Tobacco Production / Intl J Agric Biol, Vol 25, No 3, 2021

Table 5: The effect of biochar application on the large macroaggregate proportion, small macroaggregate proportion, microaggregate proportion and Silt-clay proportion in 0-20 and 20-40 cm depth

\begin{tabular}{|c|c|c|c|c|c|c|c|c|c|c|c|c|c|}
\hline \multirow[t]{2}{*}{ Depth $(\mathrm{cm})$} & \multirow[t]{2}{*}{$\begin{array}{l}\text { Biochar levels } \\
\left(\mathrm{kg} \mathrm{ha}^{-1}\right)\end{array}$} & \multicolumn{3}{|c|}{$\begin{array}{l}\text { Large macroaggregate } \\
\text { proportion (LMP) (\%) }\end{array}$} & \multicolumn{3}{|c|}{$\begin{array}{l}\text { Small macroaggregate } \\
\text { proportion (SMP) (\%) }\end{array}$} & \multicolumn{3}{|c|}{$\begin{array}{c}\text { Microaggregates proportion } \\
\text { (MIP) }(\%)\end{array}$} & \multicolumn{3}{|c|}{$\begin{array}{c}\text { Silt-clay proportion (SCP) } \\
(\%)\end{array}$} \\
\hline & & Midu & Eryuan & Jianchuan & Midu & Eryuan & Jianchuan & Midu & Eryuan & Jianchuan & Midu & Eryuan & Jianchuan \\
\hline \multirow[t]{6}{*}{$0-20$} & 0 & $24.9 \mathrm{i}$ & $26.7 \mathrm{hi}$ & $28.5 \mathrm{f}-\mathrm{i}$ & $40.1 \mathrm{~d}-\mathrm{f}$ & $37.2 \mathrm{fg}$ & $33.0 \mathrm{~h}$ & $9.1 \mathrm{~b}-\mathrm{d}$ & $11.1 \mathrm{a}$ & $10.5 \mathrm{ab}$ & $26.0 \mathrm{ab}$ & $25.1 \mathrm{ab}$ & $28.1 \mathrm{a}$ \\
\hline & 3000 & $26.5 \mathrm{hi}$ & $30.0 \mathrm{e}-\mathrm{h}$ & $31.2 \mathrm{e}-\mathrm{g}$ & $43.0 \mathrm{~b}-\mathrm{d}$ & $39.6 \mathrm{~d}-\mathrm{g}$ & $35.8 \mathrm{gh}$ & $8.1 \mathrm{c}-\mathrm{f}$ & $9.9 \mathrm{a}-\mathrm{c}$ & $9.5 \mathrm{a}-\mathrm{d}$ & $22.4 \mathrm{~b}-\mathrm{d}$ & $20.5 \mathrm{c}-\mathrm{e}$ & $23.6 \mathrm{bc}$ \\
\hline & 4500 & $27.8 \mathrm{~g}-\mathrm{i}$ & $31.7 \mathrm{e}-\mathrm{g}$ & $32.6 \mathrm{c}-\mathrm{f}$ & $45.2 \mathrm{ab}$ & $41.6 \mathrm{~b}-\mathrm{e}$ & $38.4 \mathrm{e}-\mathrm{g}$ & $7.9 \mathrm{~d}-\mathrm{f}$ & $8.8 \mathrm{~b}-\mathrm{d}$ & $8.5 \mathrm{c}-\mathrm{e}$ & $19.2 \mathrm{~d}-\mathrm{f}$ & $17.9 \mathrm{e}-\mathrm{g}$ & $20.4 \mathrm{c}-\mathrm{e}$ \\
\hline & 6000 & $29.3 \mathrm{f}-\mathrm{h}$ & $31.9 \mathrm{~d}-\mathrm{g}$ & $36.0 \mathrm{a}-\mathrm{d}$ & $47.4 \mathrm{a}$ & 43.2 b-d & $40.8 \mathrm{c}-\mathrm{f}$ & $6.9 \mathrm{e}-\mathrm{g}$ & $8.2 \mathrm{c}-\mathrm{f}$ & $6.9 \mathrm{e}-\mathrm{g}$ & $16.4 \mathrm{f}-\mathrm{h}$ & $16.7 \mathrm{e}-\mathrm{h}$ & $16.3 \mathrm{f}-\mathrm{h}$ \\
\hline & 9000 & $30.2 \mathrm{e}-\mathrm{h}$ & $33.7 \mathrm{~b}-\mathrm{e}$ & $37.5 \mathrm{ab}$ & $48.0 \mathrm{a}$ & $44.9 \mathrm{a}-\mathrm{c}$ & $41.9 \mathrm{~b}-\mathrm{e}$ & $5.7 \mathrm{~g}$ & $6.8 \mathrm{e}-\mathrm{g}$ & $6.5 \mathrm{fg}$ & $16.1 \mathrm{f}-\mathrm{h}$ & $14.7 \mathrm{gh}$ & $14.1 \mathrm{gh}$ \\
\hline & 12000 & $31.0 \mathrm{e}-\mathrm{g}$ & $36.2 \mathrm{a}-\mathrm{c}$ & $38.0 \mathrm{a}$ & $48.5 \mathrm{a}$ & $45.3 \mathrm{ab}$ & $42.5 \mathrm{~b}-\mathrm{d}$ & $5.4 \mathrm{~g}$ & $5.9 \mathrm{~g}$ & $6.5 \mathrm{fg}$ & $15.1 \mathrm{gh}$ & $12.6 \mathrm{~h}$ & $13.0 \mathrm{~h}$ \\
\hline \multirow[t]{6}{*}{$20-40$} & 0 & $31.8 \mathrm{gh}$ & $25.1 \mathrm{i}$ & $33.4 \mathrm{fg}$ & $31.8 \mathrm{e}-\mathrm{g}$ & $27.2 \mathrm{hi}$ & $20.7 \mathrm{j}$ & $10.0 \mathrm{~cd}$ & $16.8 \mathrm{a}$ & $14.4 \mathrm{~b}$ & $26.5 \mathrm{bc}$ & $31.0 \mathrm{a}$ & $31.5 \mathrm{a}$ \\
\hline & 3000 & $36.5 \mathrm{ef}$ & $27.9 \mathrm{hi}$ & $37.5 \mathrm{~d}-\mathrm{f}$ & $32.9 \mathrm{~d}-\mathrm{f}$ & $31.9 \mathrm{e}-\mathrm{g}$ & $23.3 \mathrm{ij}$ & $8.4 \mathrm{~d}-\mathrm{f}$ & $13.7 \mathrm{~b}$ & $10.9 \mathrm{c}$ & $22.2 \mathrm{~d}-\mathrm{f}$ & $26.6 \mathrm{bc}$ & $28.3 \mathrm{ab}$ \\
\hline & 4500 & 38.9 c-e & $29.1 \mathrm{hi}$ & $40.0 \mathrm{~b}-\mathrm{e}$ & $35.0 \mathrm{~b}-\mathrm{e}$ & $34.8 \mathrm{c}-\mathrm{e}$ & $28.2 \mathrm{gh}$ & $7.9 \mathrm{e}-\mathrm{g}$ & $11.0 \mathrm{c}$ & $7.6 \mathrm{fg}$ & $18.2 \mathrm{gh}$ & $25.1 \mathrm{~b}-\mathrm{d}$ & $24.0 \mathrm{~cd}$ \\
\hline & 6000 & $40.2 \mathrm{~b}-\mathrm{e}$ & $30.9 \mathrm{gh}$ & $41.9 \mathrm{bc}$ & $37.3 \mathrm{a}-\mathrm{c}$ & $36.5 \mathrm{a}-\mathrm{d}$ & $28.5 \mathrm{gh}$ & $6.3 \mathrm{~g}-\mathrm{i}$ & $9.8 \mathrm{c}-\mathrm{e}$ & $6.5 \mathrm{gh}$ & $16.2 \mathrm{~g}-\mathrm{i}$ & $22.8 \mathrm{c}-\mathrm{e}$ & $23.5 \mathrm{c}-\mathrm{e}$ \\
\hline & 9000 & 41.4 b-d & $33.9 \mathrm{fg}$ & $44.0 \mathrm{ab}$ & $39.1 \mathrm{ab}$ & $39.6 \mathrm{a}$ & $29.8 \mathrm{f}-\mathrm{h}$ & $5.1 \mathrm{hi}$ & $7.9 \mathrm{e}-\mathrm{g}$ & $6.5 \mathrm{~g}-\mathrm{i}$ & $14.3 \mathrm{~h}-\mathrm{j}$ & $18.5 \mathrm{fg}$ & $19.8 \mathrm{e}-\mathrm{g}$ \\
\hline & 12000 & $43.0 \mathrm{a}-\mathrm{c}$ & $36.4 \mathrm{ef}$ & $46.3 \mathrm{a}$ & $40.2 \mathrm{a}$ & $40.5 \mathrm{a}$ & 33.9 c-e & $4.6 \mathrm{i}$ & $6.3 \mathrm{~g}-\mathrm{i}$ & $6.3 \mathrm{f}-\mathrm{h}$ & $12.2 \mathrm{j}$ & $16.9 \mathrm{~g}-\mathrm{i}$ & $13.2 \mathrm{ij}$ \\
\hline
\end{tabular}

Means with different lowercase letters, within a column and rows for each trait, are statistically different from each other at $P<0.05$ according to DNMR test

Table 6: Effects of biochar application rates on soil organic carbon stock (SOC) and total soil nitrogen stock (STNs) in 0-20 and $20-40 \mathrm{~cm}$ depth

\begin{tabular}{|c|c|c|c|c|c|c|c|}
\hline \multirow[t]{2}{*}{ Depth $(\mathrm{cm})$} & \multirow[t]{2}{*}{ Biochar levels $\left(\mathrm{kg} \mathrm{ha}^{-1}\right)$} & \multicolumn{3}{|c|}{$\operatorname{SOCs}\left(\mathrm{g} \mathrm{C}^{2} \mathrm{~m}^{2}\right)$} & \multicolumn{3}{|c|}{ TSNs $\left(\mathrm{g} \mathrm{N} / \mathrm{m}^{2}\right)$} \\
\hline & & Midu & Eryuan & Jianchuan & Midu & Eryuan & Jianchuan \\
\hline \multirow[t]{5}{*}{$0-20$} & 0 & $3628.9 \mathrm{i}$ & $4194.4 \mathrm{~h}$ & $3803.7 \mathrm{i}$ & $324.8 \mathrm{~g}$ & $382.2 \mathrm{e}$ & $212.3 \mathrm{i}$ \\
\hline & 3000 & $5070.1 \mathrm{~d}-\mathrm{f}$ & $4963.1 \mathrm{~d}-\mathrm{f}$ & $4250.6 \mathrm{~h}$ & $451.0 \mathrm{~cd}$ & $411.0 \mathrm{~d}$ & $300.4 \mathrm{~h}$ \\
\hline & 6000 & $5266.1 \mathrm{~b}-\mathrm{d}$ & $5283.6 \mathrm{~b}-\mathrm{d}$ & $4399.1 \mathrm{gh}$ & $510.9 b$ & $479.2 \mathrm{~b}$ & $378.1 \mathrm{ef}$ \\
\hline & 9000 & $5290.3 \mathrm{~b}-\mathrm{d}$ & $5540.8 \mathrm{ab}$ & $4769.0 \mathrm{ef}$ & $551.7 \mathrm{~b}$ & $529.3 \mathrm{~b}$ & $433.8 \mathrm{~cd}$ \\
\hline & 12000 & $5440.7 \mathrm{a}-\mathrm{c}$ & $5715.6 \mathrm{a}$ & $4712.5 \mathrm{fg}$ & $532.8 \mathrm{~b}$ & $572.8 \mathrm{a}$ & $457.7 \mathrm{c}$ \\
\hline \multirow[t]{6}{*}{$20-40$} & 0 & $3466.7 \mathrm{f}-\mathrm{h}$ & $2892.3 \mathrm{i}$ & $2891.5 \mathrm{i}$ & $304.3 \mathrm{de}$ & $212.0 \mathrm{f}$ & $101.6 \mathrm{~h}$ \\
\hline & 3000 & $4249.4 \mathrm{~cd}$ & $3316.1 \mathrm{~h}$ & $3317.1 \mathrm{~h}$ & $400.8 \mathrm{c}$ & $257.6 \mathrm{e}$ & $145.7 \mathrm{~h}$ \\
\hline & 4500 & $4461.2 \mathrm{bc}$ & $3388.5 \mathrm{~h}$ & $3425.1 \mathrm{gh}$ & $457.6 \mathrm{bc}$ & $269.0 \mathrm{e}$ & $191.8 \mathrm{~g}$ \\
\hline & 6000 & $4522.6 \mathrm{a}-\mathrm{c}$ & $3411.2 \mathrm{~h}$ & $3207.1 \mathrm{hi}$ & $441.2 \mathrm{bc}$ & $269.5 \mathrm{e}$ & $160.9 \mathrm{gh}$ \\
\hline & 9000 & $4793.9 \mathrm{ab}$ & $3770.5 \mathrm{e}-\mathrm{g}$ & $3517.4 \mathrm{f}-\mathrm{h}$ & $480.4 \mathrm{ab}$ & $341.2 \mathrm{~d}$ & $261.4 \mathrm{e}^{-}$ \\
\hline & 12000 & $4836.0 \mathrm{a}$ & $3816.7 \mathrm{ef}$ & $3940.1 \mathrm{de}$ & $482.0 \mathrm{a}$ & $349.9 \mathrm{~d}$ & $333.8 \mathrm{~d}$ \\
\hline
\end{tabular}

Means with different lowercase letters, within a column and rows for each trait, are statistically different from each other at $P<0.05$ according to DNMR test

Table 7: Effects of different biochar application rates on plant height, stem circumference, maximum leaf length, and maximum leaf width at rosette stage of flue-cured tobacco grown at different sites

\begin{tabular}{|c|c|c|c|c|c|c|c|c|c|c|c|c|}
\hline \multirow[t]{2}{*}{ Biochar levels $\left(\mathrm{kg} \mathrm{ha}^{-1}\right)$} & \multicolumn{3}{|c|}{ Plant height $(\mathrm{cm})$} & \multicolumn{3}{|c|}{ Stalk girth $(\mathrm{cm})$} & \multicolumn{3}{|c|}{ Maximum leaf length $(\mathrm{cm})$} & \multicolumn{3}{|c|}{ Maximum leaf width $(\mathrm{cm})$} \\
\hline & Midu & Eryuan & Jianchuan & Midu & Eryuan & Jianchuan & Midu & Eryuan & Jianchuan & Midu & Eryuan & Jianchuan \\
\hline 0 & $7.0 \mathrm{e}$ & $7.0 \mathrm{e}$ & $7.1 \mathrm{e}$ & $4.3 \mathrm{~d}$ & $4.1 \mathrm{e}$ & $3.1 \mathrm{~g}$ & $26.0 \mathrm{~g}$ & $30.8 \mathrm{~b}$ & $21.5 \mathrm{k}$ & $12.4 \mathrm{~h}$ & $14.5 \mathrm{ef}$ & $10.7 \mathrm{j}$ \\
\hline 3000 & $7.0 \mathrm{e}$ & $7.5 \mathrm{~d}$ & $7.0 \mathrm{e}$ & $4.3 \mathrm{c}$ & $4.3 \mathrm{~d}$ & $3.0 \mathrm{~h}$ & $26.2 \mathrm{~g}$ & $31.7 \mathrm{a}$ & $21.9 \mathrm{jk}$ & $12.8 \mathrm{~g}$ & $16.3 \mathrm{~b}$ & $10.1 \mathrm{k}$ \\
\hline 4500 & $7.0 \mathrm{e}$ & $7.5 \mathrm{~d}$ & $7.0 \mathrm{e}$ & $2.7 \mathrm{i}$ & $4.4 \mathrm{bc}$ & $3.0 \mathrm{~h}$ & $26.0 \mathrm{~g}$ & $30.7 \mathrm{~b}$ & $23.9 \mathrm{~h}$ & $11.3 \mathrm{i}$ & $15.2 \mathrm{~d}$ & $12.1 \mathrm{~h}$ \\
\hline 6000 & $10.0 \mathrm{~b}$ & $11.5 \mathrm{a}$ & $10.0 \mathrm{~b}$ & $4.8 \mathrm{a}$ & $3.2 \mathrm{~g}$ & $3.0 \mathrm{~h}$ & $30.0 \mathrm{c}$ & 28.0 ef & $22.2 \mathrm{ij}$ & $15.9 \mathrm{c}$ & $17.0 \mathrm{a}$ & $11.5 \mathrm{i}$ \\
\hline 9000 & $5.5 \mathrm{~g}$ & $6.5 \mathrm{f}$ & $5.6 \mathrm{~g}$ & $4.0 \mathrm{e}$ & $4.4 \mathrm{c}$ & $2.7 \mathrm{i}$ & $29.0 \mathrm{~d}$ & $27.5 \mathrm{f}$ & 20.91 & $14.6 \mathrm{e}$ & $14.2 \mathrm{f}$ & $11.2 \mathrm{i}$ \\
\hline 12000 & $8.5 \mathrm{c}$ & $8.5 \mathrm{c}$ & $8.5 \mathrm{c}$ & $3.8 \mathrm{f}$ & $4.5 \mathrm{~b}$ & $3.2 \mathrm{~g}$ & $28.1 \mathrm{e}$ & $30.8 \mathrm{~b}$ & $22.6 \mathrm{i}$ & $14.7 \mathrm{e}$ & $15.5 \mathrm{~d}$ & $11.3 \mathrm{i}$ \\
\hline
\end{tabular}

Means with different lowercase letters, within a column and rows for each trait, are statistically different from each other at $P<0.05$ according to DNMR test

significantly higher than in Midu and Jianchuan. The difference of stem girth among the three sites was significant, with the order of Midu > Eryuan > Jianchuan. The stem girth (SG) decreased significantly compared to the control in each site when the biochar rate was 4,500 (Midu), 6,000 (Eruyuan), and 9, 000 (Jianchuan) $\mathrm{kg} \mathrm{ha}^{-1}$ respectively. The maximum leaf length and maximum leaf width of flue-cured tobacco in Midu decreased significantly at the $4,500 \mathrm{~kg} \mathrm{ha}^{-1}$ biochar rate, while in the Eryuan and Jianchuan sites they began to decrease at rates of $9,000 \mathrm{~kg} \mathrm{ha}^{-1}$. The maximum leaf length and maximum leaf width of the three sites were also significant in the order of Eryuan > Midu > Jianchuan.

\section{Effects of biochar on flue-cured tobacco yield}

When the biochar rate was 6,000 and $12,000 \mathrm{~kg} \mathrm{ha}^{-1}$, the yield of flue-cured tobacco in Midu significantly increased, by $280-300 \mathrm{~kg} \mathrm{ha}^{-1}$ compared to the control (Table 8 ). Under the same biochar application rate, the yield in Jianchuan was significantly less than in Midu and Eryuan. Similar to the yield, there was a maximum value of the proportion of superior leaves at 6,000 and $3,000 \mathrm{~kg} \mathrm{ha}^{-1}$ biochar application rates in Midu and Eryuan, respectively. In Jianchuan, the maximum value of the superior leaves appeared when the biochar application rate was $4,500 \mathrm{~kg} \mathrm{ha}^{-1}$. Except for the application rate of 6,000 and $9,000 \mathrm{~kg} \mathrm{ha}^{-1}$, the proportion of 
Li et al. / Intl J Agric Biol, Vol 25, No 3, 2021

Table 8: Effects of different biochar rates yield, proportion of superior leaves and average leaves price of tobacco grown at different locations

\begin{tabular}{|c|c|c|c|c|c|c|c|c|c|}
\hline \multirow[t]{2}{*}{ Biochar levels $\left(\mathrm{kg} \mathrm{ha}^{-1}\right)$} & \multicolumn{3}{|c|}{ Yield $\left(\mathrm{kg} \mathrm{ha}^{-1}\right)$} & \multicolumn{3}{|c|}{ Proportions of superior leaves (\%) } & \multicolumn{3}{|c|}{ Average price (dollar kg ${ }^{-1}$ ) } \\
\hline & Midu & Eryuan & Jianchuan & Midu & Eryuan & Jianchuan & Midu & Eryuan & Jianchuan \\
\hline 0 & 2892 b-d & $2853 \mathrm{c}-\mathrm{e}$ & $2533 \mathrm{gh}$ & $58.6 \mathrm{~d}$ & $53.7 \mathrm{~g}$ & $49.8 \mathrm{k}$ & $4.3 \mathrm{~d}$ & $3.7 \mathrm{i}$ & $3.6 \mathrm{j}$ \\
\hline 3000 & $2890 \mathrm{~b}-\mathrm{d}$ & 2923 bc & $2781 \mathrm{de}$ & $59.0 \mathrm{~cd}$ & $58.8 \mathrm{~d}$ & $49.3 \mathrm{k}$ & $4.5 \mathrm{~b}$ & $4.3 \mathrm{~d}$ & $4.0 \mathrm{~g}$ \\
\hline 6000 & $3174 \mathrm{a}$ & 2910 b-d & $2533 \mathrm{gh}$ & $61.8 \mathrm{a}$ & $58.5 \mathrm{~d}$ & $50.0 \mathrm{jk}$ & $4.5 \mathrm{c}$ & $4.3 \mathrm{e}$ & $3.6 \mathrm{j}$ \\
\hline 9000 & 2750 ef & 2922 bc & $2488 \mathrm{~h}$ & $57.2 \mathrm{e}$ & $56.4 \mathrm{e}$ & $51.2 \mathrm{hi}$ & $4.2 \mathrm{f}$ & $4.2 \mathrm{e}$ & $3.5 \mathrm{k}$ \\
\hline 12000 & 3193 a & $2780 \mathrm{de}$ & $2635 \mathrm{fg}$ & $61.0 \mathrm{ab}$ & $54.7 \mathrm{fg}$ & $50.9 \mathrm{ij}$ & $4.6 \mathrm{a}$ & $3.9 \mathrm{~h}$ & $3.7 \mathrm{i}$ \\
\hline
\end{tabular}

superior leaves in each site was significant with the order: Midu > Eryuan > Jianchuan. The trend in the average price was the same as that of the proportions of superior leaves.

\section{Discussion}

Soil structure plays an important role in plant growth and soil water movement, but also has a vital role in improving soil physical and chemical properties, and biological processes (Wei et al. 2006; An et al. 2008). In this experiment, biochar application decreased the soil bulk density (Table 4), was due to the porous structure of biochar, which effectively increase soil porosity and improve soil aeration. This result is similar to the results of Meng et al. (2020). The mean weight diameter and geometric mean diameter of soil aggregates increased with the biochar application rate in each site, indicating that biochar application could improve soil aggregate stability; these results are consistent with Zhu et al. (2018). The MWD and GMD at Eryuan were significantly lower than at Midu and Jianchuan for the $20-40 \mathrm{~cm}$ depth (Table 4). We believe the main reason for this difference among the three sites was the variation of soil nutrients (Shinjo et al. 2000). Compared with Midu and Jianchuan, the soil organic matter and total nitrogen content in Eryuan were significantly less than in the other two sites. This limited the availability of nutrients to microorganisms in soil, and because the secretion of microbial products and the production of hyphae are the core of soil aggregation, this limited the increase of aggregate mean weight diameter and geometric mean diameter (Wang et al. 2020).

Many studies show that the quantity and distribution of water stable aggregates determine the stability of soil structure and its resistance to erosion, especially the number of aggregates with particle size $>0.25 \mathrm{~mm}$, which is one of the important indexes to determine the quality of soil ( $\mathrm{Li} e t$ al. 2014; Amundson et al. 2015). Applying biochar significantly increased the proportion of large and small aggregates and decreased the proportion of microaggregates and silty clay -sized aggregates. This result was similar with previous studies (Chen et al. 2008). The main reason for the change in aggregate proportion was that the biochar having a nutrient holding capacity effect, offering sufficient nutrient for the growth and reproduction of microorganisms. The hyphae of fungi and actinomycetes can mechanically
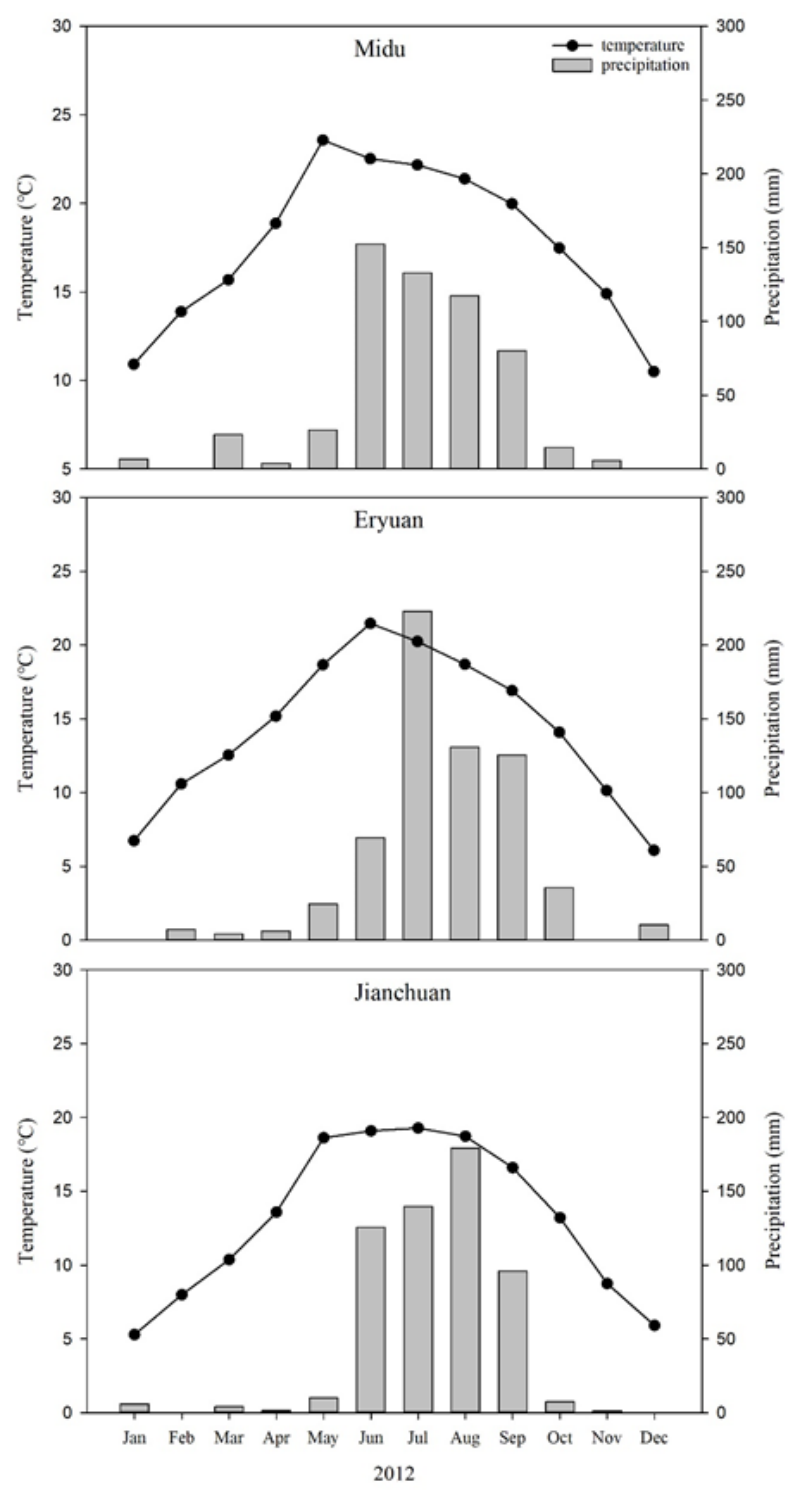

Fig. 1: The average monthly temperature and monthly accumulated precipitation at Midu, Eryuan and Jianchuan

entangle particles in the soil to form aggregates, thus increasing the proportion of large and small aggregates. However, the proportion of large aggregates in Jianchuan was significantly higher than the other two sites (Table 5). 
This may be related to the difference of precipitation and temperature in three sites (Fig. 1). Previous studies showed that soil aggregate stability was negatively correlated with temperature and soil moisture. High temperature and high humidity will reduce soil aggregate stability (Ye et al. 2013; $\mathrm{Xu}$ et al. 2019). In this study, the temperature and precipitation in Jianchuan during soil sampling period (Apr. to Sep.) were lower than in Midu and Eryuan (Fig. 1), which was conducive to maintaining the activity of soil microorganisms and the stability of soil structure. It was beneficial to the formation of large aggregates.

Many studies have shown that biochar can significantly enlarge the SOCs and TSNs in topsoil (Shinjo et al. 2000; Mao et al. 2008). As an exogenous organic matter, biochar can directly increase the soil organic matter content and maintain the relative stability of soil organic carbon stocks (Wang and Sun 2016). The present study showed that biochar application could significantly enlarge soil SOCs (Midu) and TSNs (Jianchuan). It is consistent with the results of previous studies (Wang and Sun 2016). Ji et al. (2018) hold the opinion that adding high $\mathrm{C} / \mathrm{N}$ ratio biochar into low organic matter soil or adding low activity biochar in high organic matter soil could inhibit the mineralization of soil organic matter. In this study, the soil organic matter content in Midu was highest. Adding biochar could inhibit organic matter mineralization in soil, thus contributing to the stability of SOCs. However, the content of soil organic matter, animal, plant, and microbial residues in $20-40 \mathrm{~cm}$ depth are less than topsoil, which makes its storage capacity smaller.

Plants growth depends on good soil environment. The plant height increased significantly when the biochar rate was $6000 \mathrm{~kg} \mathrm{ha}^{-1}$. This result is consistent with previous studies (Chen and Du 2015; Minhas et al. 2020). However, when the biochar rate was $9000 \mathrm{~kg} \mathrm{ha}^{-1}$, the plant height decreased significantly compared with no biochar application. That is because biochar has a threshold effect (Zhang et al. 2015). When the biochar application rate was low, it provided tobacco with nutrients for the growth; when the rate was high, the soil structure was destroyed, and the crop growth affected negatively (Wei et al. 2019). Excessive carbon accumulated in the soil will affect the absorption of water and nutrients by plant roots, resulting in the decrease of yield and its components (Liang et al. 2006; Qiu et al. 2020). The stalk girth at a certain biochar concentration decreased compared with the control (Table 7), which may be related to nitrogen leaching caused by biochar. With benefits from the improvement of soil physical and chemical properties, the yield of tobacco leaves and the proportion of upper leaves also increased, which contributed to the increase of average price.

Previous research showed that after applying biochar, the availability of soil nutrients and the efficiency of plant nutrient absorption were improved (Jeffery et al. 2011). In this study, the effects of biochar on yield and quality of fluecured tobacco in different locations were in the order of
Midu > Eryuan > Jianchuan. This is mainly because at the rosette stage (late May to early June), the Midu site had the highest SOM, total nitrogen content and total soil nitrogen stock among three sites, which provided sufficient nutrition for the tobacco roots growth. Moreover, the precipitation in Midu area is less than that in Eryuan site, which reduces the nitrogen leaching in topsoil (Table 6).

\section{Conclusion}

Tobacco stalk biochar improved soil physical structure. Biochar decreased soil bulk density, increased the proportion and diameter of large and small soil aggregates, and increased soil organic carbon and nitrogen stocks. Biochar addition also improved the yield and quality of tobacco leaves. However, if the biochar application rate exceeded 9,000 $\mathrm{kg} \mathrm{ha}^{-1}$, it deteriorated conditions conducive to plant growth. The optimum biochar application rates for better plant height, stem diameter, maximum leaf length and leaf width, yield, and average price differed by site. These rates were: $6,000 \mathrm{~kg} \mathrm{ha}^{-1}$ (Midu), 3,000 $\mathrm{kg} \mathrm{ha}^{-1}$ (Eryuan) and $4,500 \mathrm{~kg} \mathrm{ha}^{-1}$ (Jianchuan), respectively.

\section{Acknowledgements}

The authors are thankful to Mark S. Coyne for his valuable assistance and advice in the preparation of this paper. This work was financially supported in part by the Yunnan Fundamental Research Project (grant NO. 2017FB074, 202001AT070013) and the National Natural Science Foundation of China Grant (41601330), and the Yunnan Provincial Tobacco Monopoly Bureau Grants (2017YN10, 2017YN09, 2017YN07, 2018530000241017, 2020530000241025 and 2019530000241019). Authors thank to Yunnan Technology Innovation Program (2019HB068) and Yunnan Ten Thousand People Program (YNWR-QNBJ-2018-400) for supporting Congming Zou.

\section{Author Contributions}

Y Li and CM Zou designed the study and collected the data; X He and MY Hu conceived the idea; JE Su, TY Xu and R Liu carried out the field experiment; TX Liu and $\mathrm{KY} \mathrm{Gu}$ analyzed the data; all authors contributed to the writing and revisions.

\section{References}

Amundson R, AA Berhe, JW Hopmans, C Olson, AE Sztein, DL Sparks (2015). Soil science. Soil and human security in the $21^{\text {st }}$ century. Science 348:1261071-1261079

An SS, YM Huang, FL Zheng, JG Yang (2008). Aggregate characteristics during natural revegetation on the Loess Plateau. Pedosphere 18:809-816

Baruah N, N Gogoi, M Farooq (2020) Influence of biochar and organic soil amendments on bioavailability and immobilization of copper and lead to common cocklebur in acidic sandy loam soil. $J$ Environ Chem Eng 8; Article 104480 
Chen M, XG Du (2015). Effect of biochar on soil properties and yield and quality of tobacco. Chin Soils Fert 01:80-83

Chen Y, Y Yan, ML Zhang, HB He, HT Xie, XD Zhang, P Zhu (2008). Effect of long-term fertilization on aggregation and carbon and nitrogen contents in malison. Chin J Soil Sci 6:1288-1292

Cheng HY, ZW Xiao, JH Qin, R Wang (2013). Effects of continuous cropping on soil nutrient and enzyme activities of corn seed field soil. Soils 45:623-627

El-Naggar A, SS Lee, J Rinklebed, M Farooq, AK Sarmah, AR Zimmerman, M Ahmad, SM Shaheen, YS Ok (2019) Biochar application to low fertility soils: A review of current status, and future prospects. Geoderma 337:536-554

Emma M (2006). Putting the carbon back: Black is the new green. Nature 442:624-626

Farooq M, L Romdhane, A Rehman, AKM Al-Alawi, WM Al-Busaidi, SA Asad, D-J Lee (2020a) Integration of seed priming and biochar application improves drought tolerance in cowpea. J Plant Growth Regul doi: 10.1007/s00344-020-10245-7; In press

Farooq M, A Rehman, AKM Al-Alawi, WM Al-Busaidi, D-J Lee (2020c) Integrated use of seed priming and biochar improves salt tolerance in cowpea. Sci Hort 272: 109507

Farooq M, A Ullah, M Usman, KHM Siddique (2020b) Application of zinc and biochar help to mitigate cadmium stress in bread wheat raised from seeds with high intrinsic zinc. Chemosphere 260:127652

Han F, R Wang (2016). Status quo and development strategy of green development of tobacco stalks organic fertilizer industrialization. Acta Tab Sin 22:126-132

Hussain M, M Farooq, A Nawaz, AM Al-Sadi, ZM Solaiman, SS Alghamdi, U Ammara, YS Ok, KHM Siddique (2017). Biochar for crop production: Potential benefits and risks. J Soils Sedim 17:685-716

Jeffery S, FGA Verheijen, MVD Velde, AC Bastos (2011). A quantitative review of the effects of biochar application to soils on crop productivity using meta-analysis. Agric Ecosyst Environ 144:175-187

Ji GF, PZ Wang, SW Zhang, YX Xing, JZ Li, HY Jiang (2018). Effects of biochar on $\mathrm{C} / \mathrm{N}$ value of cinnamon soil in tobacco growing areas and the root growth of flue-cured tobacco. J Henan Agric Univ 52:863-867

Li FY, JG Tao, JF Wang, XL Li (2017). Difference characteristics of biochar derived from peanut shell under different temperatures. Chin J Environ Eng 11:3726-3730

Li QQ, CY Xu, ZC Geng, JC Zhang, SL Chen, HL Wang, Y Zhang, FY Yun, L Yang, SH Dong (2019). Impact of biochar on soil bulk density and aggregates of Lou soil. Chin J Environ Sci 40:3388-3396

Li W, ZC Zheng, TX Li, MY Liu (2014). Distribution characteristics of soil aggregates and its organic carbon in different tea plantation age. Acta Ecol Sin 34:6326-6336

Liang BQ, J Lehmann, D Solomon, J Kinyangi, JM Grossman, B Oneill, JO Skjemstad, JE Thies, F Luizao, JB Petersen, EG Neves (2006). Black carbon increases cation exchange capacity in soils. Soil Sci Soc Amer J 70:1719-1730

Lin QH, HL Liu, L Wang, YG Hua, ZZ Cha, W Luo (2018). Effect of biochar-based soil conditioner on the soil $\mathrm{pH}$ and $\mathrm{CEC}$ in rubber plantations. Chin J Trop Agric Sci 38:1-4

Liu GZ, FG Yang, B Xu (2020). Advances in research on effects of biochar on soil nitrogen leaching. Soil Water Conserv Chin 18:139-149

Ma YY, YL Li, HX Lai, Q Guo, QH Xue (2017). Effect of sick rhizosphere soil under tomato continuous cropping on soil nematodes, microbes, and tomato growth. Chin J Eco-Agric 25:730-739

Mao YL, YS Yang, SH Xing, GQ Wang, JF Guo (2008). Effects of land use change on light fractions of organic carbon in soil water-stable aggregates. J Soil Water Conserv 22:132-137
Meng FH, XF Yu, ZG Wang, SP Hu, JY Sun, GE Qing, Q JW, JL Gao (2020). Effects of physical property of soil and yield of spring corn by combined application of biochar and nitrogen. J Maize Sci $28: 142-150$

Minhas WA, M Hussain, N Mehboob, A Nawaz, S Ul-Allah, MS Rizwan, Z Hassan (2020). Synergetic use of biochar and synthetic nitrogen and phosphorus fertilizers to improves maize productivity and nutrient retention in loamy soil. J Plant Nutr 43:1356-1368

Qin Y, K Ma, P Liu (2015). Effect of potato continuous cropping on genetic diversity of soil microorganisms. Chin J Eco-Agric 23:225-232

Qiu LJ, X Zhang, L Li, Q Li, YY Suo, PJ Cheng, XJ Si, D Chang (2020). Effects of biochar application amount on soil properties and tobacco yield and quality. J Anhui Agric Sci 48:153-156

Sarma B, M Farooq, N Gogoi, B Borkotoki, R Kataki, A Garg (2018) Soil organic carbon dynamics in wheat - green gram crop rotation amended with Vermicompost and biochar in combination with inorganic fertilizers: A comparative study. J Clean Prod 201: 471-480

Shinjo H, H Fujita, G Gintzburger (2000). Soil aggregate stability under different landscapes and vegetation types in a semiarid area in Northeastern Syria. Soil Sci Plant Nutr 46:229-240

Wang F, HW Sun (2016). Sorption mechanisms of polar and apolar organic contaminants onto biochars. Environ Chem 11:3726-3730

Wang RF, LX Zhao, YJ Shen, HB Meng, HZ Yang (2015). Research progress on preparing biochar and its effect on soil physio-chemical. J Agric Sci Technol 17:126-133

Wang X, ZK Zhong, XY Zhang, SJ Wu, XQ Lu, GH Yang, CJ Ren, XH Han (2020). Relationship between the composition of soil aggregates and the distribution of organic carbon under long-term abandoned restoration. Chin J Environ Sin 41:2416-2424

Wei CF, M Gao, JA Shao, DT Xie, GX Pan (2006). Soil aggregate and its response to land management practices. Particuology 4:211-219

Wei YX, H Wang, H Liu, Y Wu (2019). Influences of biochar on soil fertility and soybean yield and its prediction. Trans Chin Soc Agric Mach 50:302-312

Xu C, F Gu, Y Wang, YB He, JZ Chen (2019). Study on the relationships between soil aggregate and water dynamics under three vegetation cover. J Soil Water Conserv 33:68-74

Ye JS, T Li, YJ Hu, ZP Hao, YZ Gao, YS Wang, BD Chen (2013). Influences of AM fungi on plant growth and water-stable soil aggregates under drought stresses. Acta Ecol Sin 33:1080-1090

Yuan S, LX Zhao, HB Meng, YJ Shen (2016). The main types of biochar and their properties and expectation researches. Plant Nutr Fert Sci 22:1402-1417

Zhang H, Y Li, YJ Zhang, JF Zhu, GY Jiang, SL Liu, FM Shen, F Liu (2018). Biochar affects carbon and nitrogen conversion under different nitrogen application rates in tobacco-planting soil. J Agric 8:27-32

Zhang RK, TC Ma (2020). Analysis of applying pesticides among major grains in Yunnan Province in 2019. Yunnan Agric Technol 3:13-16

Zhang WM, XC Guan, YW Huang, DQ Sun, J Meng, WF Chen (2015). Biological effects of biochar and fertilizer interaction in soybean plant. Acta Agron Sin 41:109-122

Zhu Q, TT Yan, ZD Zhou, JM Sun, JH Xue, YB Wu (2018). Effects of biochar application on characteristics of karst calcareous soil and growth of Robinia pseudoacacia seedlings. Jiangsu Agric Sci 46:241-245

Zou C, RC Pearce, JH Grove, MS Coyne (2017). No-tillage culture and nitrogen fertilizer management for burley tobacco production. $J$ Agric Sci 155:599-612

Zou C, RC Pearce, JH Grove, MS Coyne (2015). Conservation practices in tobacco production increase large aggregates and associated carbon and nitrogen. Soil Sci Soc Amer J 79:1760-1770 